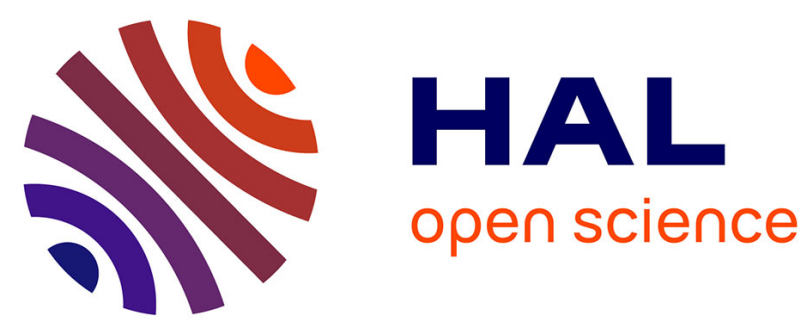

\title{
An Evolutionary Approach For Blind Deconvolution Of Barcode Images With Nonuniform Illumination
}

Laurent Dumas, Mohammed El Rhabi, Gilles Rochefort

\section{To cite this version:}

Laurent Dumas, Mohammed El Rhabi, Gilles Rochefort. An Evolutionary Approach For Blind Deconvolution Of Barcode Images With Nonuniform Illumination. 2011 IEEE Congress on Evolutionary Computation (CEC), Jun 2011, New Orleans, United States. 10.1109/CEC.2011.5949917 . hal01812543

HAL Id: hal-01812543

https://hal-enpc.archives-ouvertes.fr/hal-01812543

Submitted on 11 Jun 2018

HAL is a multi-disciplinary open access archive for the deposit and dissemination of scientific research documents, whether they are published or not. The documents may come from teaching and research institutions in France or abroad, or from public or private research centers.
L'archive ouverte pluridisciplinaire HAL, est destinée au dépôt et à la diffusion de documents scientifiques de niveau recherche, publiés ou non, émanant des établissements d'enseignement et de recherche français ou étrangers, des laboratoires publics ou privés. 


\section{An Evolutionary Approach For Blind Deconvolution Of Barcode Images With Nonuniform Illumination}

\author{
Laurent Dumas \\ Laboratoire de Mathématiques de Versailles, \\ Université de Versailles, France \\ Email: dumas@math.uvsq.fr
}

\author{
Mohammed El Rhabi \\ Université Cadi Ayyad \\ FST, Marrakech, Maroc \\ Email: elrhabi@gmail.com
}

\author{
Gilles Rochefort \\ RealEyes3D \\ F92-213, Saint Cloud, France \\ Email: grochefort@realeyes3d.com
}

\begin{abstract}
This paper deals with a joint nonuniform illumination estimation and blind deconvolution for barcode signals by using evolutionary algorithms. Indeed, such optimization problems are highly non convex and a robust method is needed in case of noisy and/or blurred signals and nonuniform illumination. Here, we present the construction of a genetic algorithm combining discrete and continuous optimization which is successfully applied to decode real images with very strong noise and blur.
\end{abstract}

\section{INTRODUCTION}

This paper is devoted to the problem of blind deconvolution of linear barcode signals. Linear barcodes are one of the oldest technology related to automatic identification and data capture. Typical barcodes symbologies are UPC/EAN/JAN and are one variation of over 250. Linear barcodes are graphical representations of a finite sequences of digits. Information is encoded in the widths (lines) and the spacings of parallel lines. Up to a choosen symbology, each sequence of digits admits a unique graphical representation. So, retrieving digits from an acquired barcode require accurate estimation of the true lenght bars and spaces pattern. In order to help decoding, some symbology may include special start/stop group of bars and a validation checksum digit.

Ideally, the barcode is given as a binary one-dimensional signal when scanned by a laser or a binary image when scanned by a camera ( 1 represents white space and 0 indicates a black bar).

Linear barcodes are optimized to be read by a laser scanner. Typically UPC, EAN, JAN symbologies have been widely adopted in industrial applications (supermarket checkout system, labelling railroad cars, ...). Nowadays, every article has its own printed barcode. One may imagine using barcodes for its own purpose, for example price comparison, allergen database (gluten, peanut ...). Unfortunately, it is not usual to have its own personal barcode readers.

So, computer vision based barcode recognition could be an interesting alternative. Here, the problem could be separated in two steps: localization and decoding. Computer approach increases the performance of both steps, barcode localization and decoding (see [1], [2]). In the computer vision approaches, low cost cameras (webcam, cameraphone, ...), without autofocusing or macro mode, could introduce some distortions and noise artifacts. As a consequence, decoding performances depend on the distance from the camera to the surface where the barcode appears ([3]).

Anyway, acquired images are often blurred and distorted by various factors, including speckle noise, ambient light. Then, a way, to decode barcodes included in an image, is first to restore it. We would like to retrieve a barcode image with blur, noise and also distorted by nonuniform illumination, that has been acquired by a low cost camera (see an example on Figure 1).

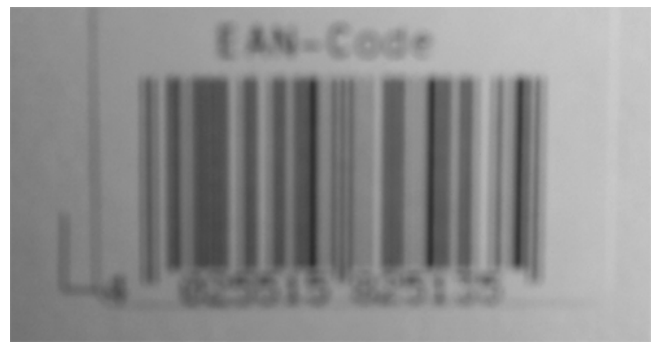

Fig. 1. A real barcode image with blur, noise and non uniform illumination

To reduce this problem, in [4], the author proposes some additional hardware device to overcome this drawback, but not convenient in the context of a mobile phone with a digital camera [5]. To consider the effect of nonuniform illumination, several methods have been proposed, among them the illumination cone method, a spherical harmonic based representation [6], quotient image based approaches [7] and correlation based method [8]. The performance of these methods are poor and many of them require either knowledge of light source or a large number of training data which is not tractable in real world applications.

Generally speaking, an image $u$ could be seen as the product of a reflectance $R$ and the illumination effect $I$ (see [9]). This model has been used in [10] to deblur barcode signals under nonuniform illumination. The blurring operator is a Gaussian function with unknown standard deviation. The solution is estimated by a penalized non linear least square objective function, based on a proper parametrization of a linear barcode and nonuniform illumination. The solution is then estimated by the well-known gradient based method.

In this paper, we propose a novel method for blind deconvolution of barcode signals in the out-of-focus case, based on a 
genetic algorithm taking into account the highly non convexity of the problem and the fact that the target solution is a binary image.

The paper is organized as follows : section II gives a mathematical definition of the barcode and presents the problem. Section III proposes a solution to the problem by means of a genetic algorithm, describes the method and shows how to implement it. Finally, some numerical results illustrate this work in Section IV.

\section{THE MATHEMATICAL MODEL}

\section{A. A mathematical definition for a barcode}

Here, we give a general definition of a barcode in the bidimensional case. We suppose that $f(x, y)$ is a $2 D$ continuous function representing the intensity value of the barcode at location $(x, y)$ :

$$
f(x, y)=\left\{\begin{array}{l}
1, \text { if }(x, y) \in B_{k} \\
0, \text { else }
\end{array}\right.
$$

where $B_{k}$ represents the region bounded by the $k^{\text {th }}$ bar. Assume that $f_{d}(i, j)$ is a discrete function acquired from $f(x, y)$ such that :

$$
f_{d}(i, j)=\int_{j}^{j+1} \int_{i}^{i+1} f(x, y) d x d y
$$

where $(i, j)=(0,0),(0,1), \ldots,(1,0), \ldots,(N, M) . f_{d}(i, j)$ is the area of the region in pixel $(i, j)$ bounded by the bar passing through that pixel, each pixel is considered as a square region.

\section{B. The blurring model}

Let $u \in \mathbb{R}^{N M}$ be an original $N \times M$ gray-scale image, and $K \in \mathbb{R}^{N M \times N M}$ represents a blurring (or convolution) operator, $I \in \mathbb{R}^{N M}$ the non uniform illumination, $n \in \mathbb{R}^{N M}$ the additive noise, and $u_{0} \in \mathbb{R}^{N M}$ an observation which satisfies the relationship:

$$
u_{0}=I K u+n,
$$

In general, the blurring operator $\mathrm{K}$ is supposed shift-invariant, and takes the following form:

$$
K=k \star u
$$

where $k$ is the point spreading function (PSF). In many real cases, the blurring operator $K$ is unknown. Usually, we assume that $K$ could be represented by few parameters $p$, these parameters are a priori unknown. For example, in the outof-focus case, the PSF could be approximated as :

$$
k(\boldsymbol{x}, \boldsymbol{y})=\frac{1}{\pi r^{2}} \mathbb{1}_{B_{r}}(\boldsymbol{x}, \boldsymbol{y})
$$

where $B_{r}$ is the ball with the center 0 and radius $r>0$ and $\mathbb{1}_{B_{r}}(\boldsymbol{x}, \boldsymbol{y})$ defines its indicator function.

The non uniform illumination, $I$, is also unknown. Generally, it is assumed smooth and could be modelled by using B-spline functions that are uniformly located in the spatial domain ([10]):

$$
I(\boldsymbol{x}, \boldsymbol{y})=\sum_{i=1}^{L_{x}} \sum_{j=1}^{L_{y}} I_{i j} \beta^{n}\left(\boldsymbol{x}-\boldsymbol{l}_{i}\right) \beta^{n}\left(\boldsymbol{y}-\boldsymbol{l}_{j}\right),
$$

where $\beta^{n}(t)$ is a $n$-order $\mathrm{B}$-spline function centered at zero, $L_{x}$ (resp. $L_{y}$ ) is the number of the B-spline functions, $I_{i j}$ the B-spline coefficients and $\boldsymbol{l}_{i}, i \in\left\{1, \ldots, L_{x}\right\}$ (resp. $\boldsymbol{l}_{j}, j \in$ $\left\{1, \ldots, L_{y}\right\}$ ) denote uniformly distributed center locations of the B-spline functions.

\section{The inverse problem}

If we suppose that $n$ is a gaussian noise, the joint estimation of the blur kernel $K$, the illumination $I$ and the restored image $u$ is an ill-posed problem. An approximation of $K$ (or $r$ equivalently), $I$ and $u$ could be obtained by solving the following non convex optimization problem:

$$
\min _{r, I, u} E(r, I, u)=\int_{\Omega}\left|I K(r) u-u_{0}\right|^{2} d x d y
$$

In order to control the noise, a regularization term of the type $\lambda \int_{\Omega} \varphi(|\nabla u|) d x d y$ must be added to the cost function $E$. Yet, the choice of the function $\varphi$ must be relevant and is often a difficult task. Moreover, the use of a deterministic descent method, such as steepest descent, is only able to seek for a local minima of the cost function, which is unfortunately highly non linear.

\section{SOLVING THE INVERSE PROBLEM}

As seen above, the solution of the inverse problem depends on the regularization term. The method of genetic algorithms is used here to overcome this drawback and to solve the inverse problem on a robust way with respect to noise and blur.

\section{A. The evolutionary algorithm}

Genetic algorithms are global optimization methods directly inspired from the Darwinian theory of evolution of species ([11]). They consist in following the evolution of a certain number $N_{p}$ of possible solutions, also called population. To each element (or individual) $x_{i} \in \mathcal{O}$ of the population is affected a fitness value inversely proportional to $J\left(x_{i}\right)$, in case of a minimization problem for the cost function $J$. The population is regenerated $N_{g}$ times by using three stochastic principles called selection, crossover and mutation, that mimics the biological law of the 'survival of the fittest'.

They have show their efficiency in many applicative fields, in engineering science or in medicine, cite for instance, car shape optimization [12], turbine shape optimization [13], pacemaker optimization [14], to mention some results obtained by one of the author.

The genetic algorithm that is used here acts in the following way: at each generation, $\frac{N_{p}}{2}$ couples are selected by using a roulette wheel process with respective parts based on the fitness rank of each individual in the population. To each selected couple, the Darwinian principles, namely crossover and mutation, are then successively applied with a respective probability $p_{c}$ and $p_{m}$. A one-elitism principle is added in 
order to be sure to keep in the population the best element of the previous generation.

\section{B. The cost function}

The cost function that has been used is different from the one written in (6) because the regularization term has been removed and the $L^{2}$ norm is replaced by the $L^{4}$ norm:

$$
\min _{r, I, u} J(r, I, u)=\int_{\Omega}\left|I K(r) u-u_{0}\right|^{4} d x d y .
$$

Here, The choice of the $L^{4}$ norm is not crucial but it has shown better convergence properties.

\section{The search space}

One key feature of genetic algorithms is their ability to deal, either with discrete or continuous search spaces, or even with both of them. It is precisely the case here as the search space for $u$ is of finite type whereas the search space for $I$ and $r$ is of continuous type:

(i) The search space for $u$

In this work, a particular symbology of barcode has been studied, namely the EAN13 type, which is currently the most used one. It encodes 13 characters by using the width of 30 vertical bars. It is worth noticing that the chosen approach can easily adapt to any other type of barcode. As shown in Figure 2 , the signal $u$ to be found is discretized in 61 integer values, gathered in a vector $U$ :

$$
U=\left(U_{1}, \ldots, U_{61}\right) \in \mathbb{N}^{61}
$$

which corresponds to the interval lengths (in terms of pixels) of each successive sequence of white and black colors (where $u$ is respectively chosen equal to 0 and 1 ).

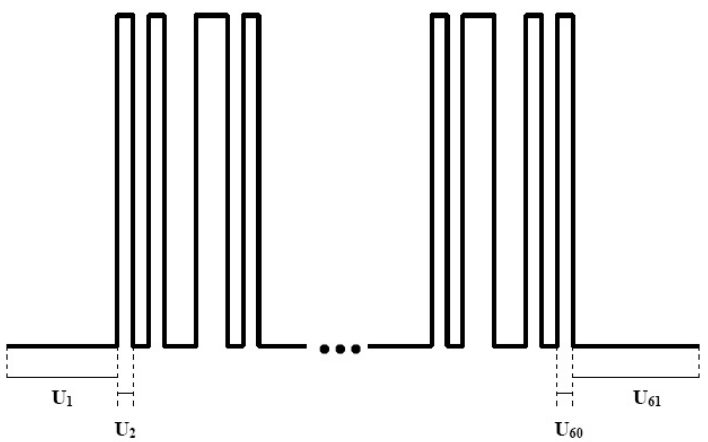

Fig. 2. The discrete search space for $u$ : example of a barcode representation

Note in particular that $U_{1}$ and $U_{61}$ play a particular role because they correspond to the left, respectively right, unknown margin at each side of the barcode in the image. Note also that the constraint

$$
\sum_{i=1}^{61} U_{i}=M
$$

where $M$ is the width of the image has to be fulfilled. (ii) The search space for $r$ and I

It is a given interval of $\mathbb{R}_{+}$for $r$ whereas it is an hypercube included in $[0,1]^{L_{x}}$, for $I$. The latter corresponds to the ordinates of $L_{x}$ points used for the reconstruction of $I$ by cubic splines interpolation (see Figure 3 for an example of a nonuniform illumination obtained from an interpolation with 5 points).

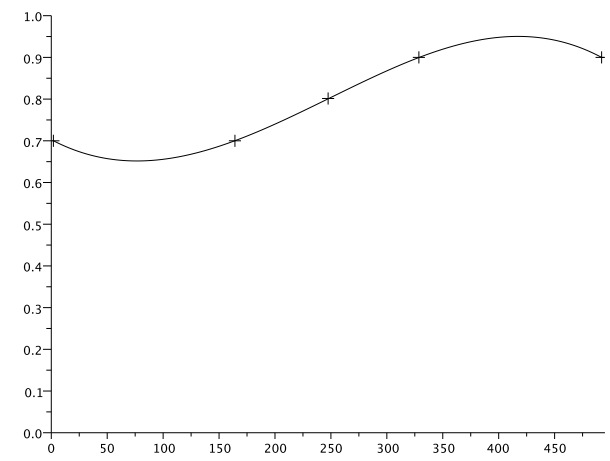

Fig. 3. The continous search space for $I$ : example of a nonuniform illumination

\section{The darwinian principles}

The darwinian principles, namely crossover and mutation, play a crucial role in the convergence properties of genetic algorithms and have to be chosen with care.

(i) The darwinian principles for the discrete variable $U$

The crossover principle between two vectors $U$ and $V$ in $\in \mathbb{N}^{61}$ is inspired from the original crossover in the case of chromosomes: it consists in creating two offsprings by exchanging a given number, $m$, of randomly chosen consecutive sequences of each vector ( $m$-point crossover). As the obtained offsprings do not generally satisfy the constraint (8), a renormalization principle is added which consists in increasing (or reducing) the value of randomly chosen components from them. Actually, this last process acts as the mutation operator.

Assume for instance that $m=1$ and denoting by $U$ and $V$ the two parents:

$$
\begin{aligned}
& U=\left(U_{1}, \ldots, U_{61}\right) \\
& V=\left(V_{1}, \ldots, V_{61}\right)
\end{aligned}
$$

then the two offsprings are respectively:

$$
\begin{aligned}
U^{\prime} & =\left(U_{1}, \ldots, U_{k}, V_{k+1} \ldots, V_{61}\right) \\
V^{\prime \prime} & =\left(V_{1}, \ldots, V_{k}, U_{k+1} \ldots, U_{61}\right)
\end{aligned}
$$

for a random $k \in\{1, \ldots, 61\}$. Then two offsprings are mutated with the iterated process

$$
U_{i}^{\prime}=U_{i}^{\prime} \pm 1 \quad \text { for a random } i \in\{1, \ldots, 61\}
$$

until

$$
\sum_{i=1}^{61} U_{i}^{\prime}=M
$$


(ii) The darwinian principles for the continuous variables

For the continuous variables $I$ and $r$, it consists in a barycentric combination for crossover and a non uniform mutation. For instance for the blur radius, considering two parents $r_{1}$ and $r_{2}$, the crossover and the mutation respectively write as:

$$
\left(r_{1}^{\prime}, r_{2}^{\prime}\right)=\left(\alpha r_{1}+(1-\alpha) r_{2}, \alpha r_{2}+(1-\alpha) r_{1}\right)
$$

and

$$
\begin{gathered}
r_{i}^{\prime \prime}=r_{i}^{\prime}+\beta\left(r_{\max }-r_{i}\right)^{b} \\
\left(\text { or } r_{i}^{\prime \prime}=r_{i}^{\prime}-\beta\left(r_{i}-r_{\text {min }}\right)^{b} \text { with probability } 0.5\right)
\end{gathered}
$$

where $\alpha$ and $\beta$ are random numbers between 0 and 1 and $b \in[1,5]$.

\section{REsults}

Four examples of results are presented here. The first two examples are validation test cases: they consist in determining in the absence of noise, the continuous (respectively discrete variables) knowing the other ones. The third example is a complete deconvolution case of an image without knowing any information (illumination, blur, position of the barcode in the image, etc...) and with a strong noise. Finally the fourth example is an example of a barcode decoding from a real image, namely the one depicted in Figure 1.

Example 1: determination of blur radius and nonuniform illumination of an image with a known barcode, no noise

In this first example, the only unknowns are the blur radius and the nonuniform illumination function of an image of a known barcode. An evolutionary algorithm with 200 individuals and 150 generations is always able to approximate very accurately these two quantities. Actually, a quasi perfect agreement is achieved for the illumination function, as well as for the blur radius, in any configuration showing the robustness of the approach. For example, a comparison is made on Figure 4 between the original illumination (full line), the initial best illumination (dotted line) and the final best one after the optimization process (mixed dotted line, almost superimposed to the original illumination).

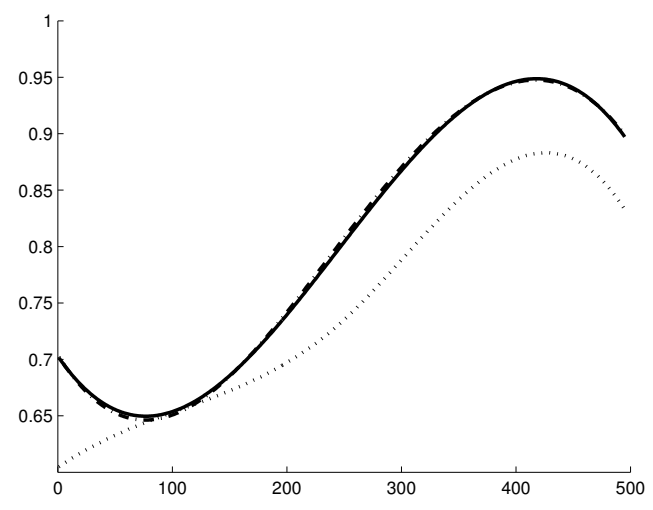

Fig. 4. The exact illumination, the final best illumination (almost superimposed) and the initial best illumination (dotted line)
Example 2: determination of a barcode characteristics with a known blur, no illumination, no noise

The objective is here to show that the evolutionary algorithm acting only on the discrete variables, that is the vector $U \in \mathbb{N}^{61}$, is able to find an exact barcode corresponding to a given vector $U_{0} \in \mathbb{N}^{61}$. In this case, the blur radius $r=3$ is fixed and the unknowns are the 61 coordinates of $U$. With a population number of 200 individuals and a generation number around 300 , the algorithm always achieves a perfect convergence, even starting with a very bad initial guess (see Figure 5 for an example).

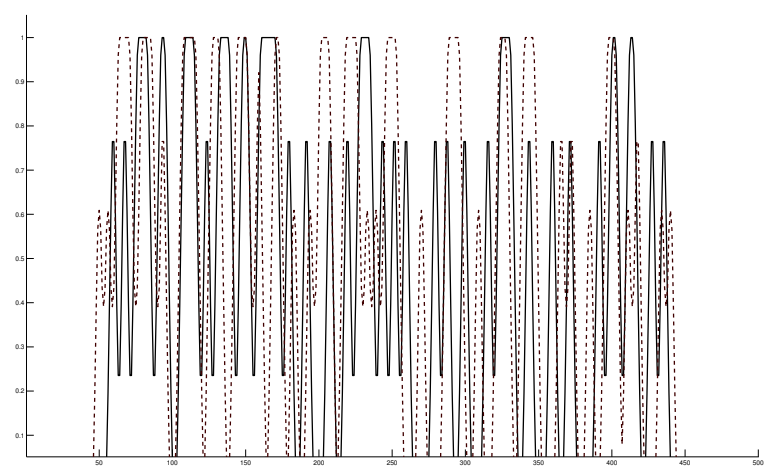

Fig. 5. The exact signal, the final best signal (almost superimposed) and the initial best signal (dotted line)

Example 3: determination of a barcode characteristics without any information on blur, illumination and noise on the image An example of blind deconvolution of a barcode signal is presented here. It corresponds to the deblurring of the signal $u_{0}$ defined on 494 pixels and depicted in Figure 6.

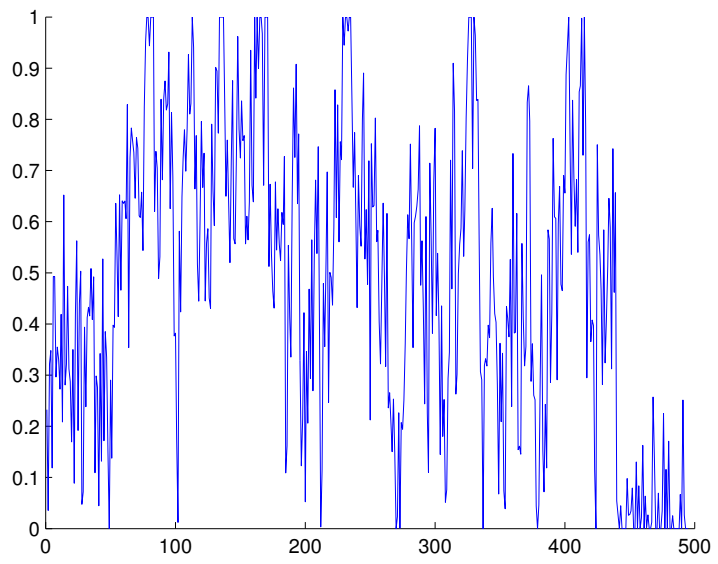

Fig. 6. The observed signal $u_{0}$

As it can be observed, it is an example of a signal, with a strong noise and blur and with a nonuniform illumination. It is representative of the robustness and accuracy of our algorithm 
for deblurring and denoising. Obviously, the same kind of results can be reproduced with very similar signal.

The evolutionary algorithm presented in the previous section is applied with the following parameters:

$$
\left(N_{p}, N_{g}, p_{c}, p_{m}\right)=(5000,350,0.8,0.5)
$$

Concerning the search domain, no hypothesis is made on the localization of the barcode in the image. Value of the out of focus radius is supposed to lie between values 2 and 7 .

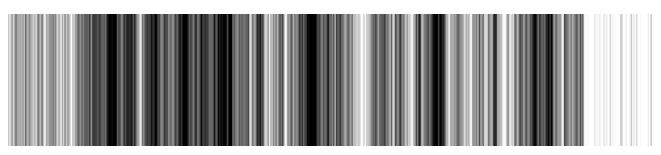

(a) The observed barcode

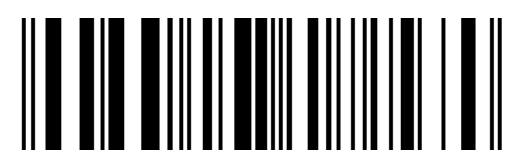

(b) The initial best barcode (not decodable)

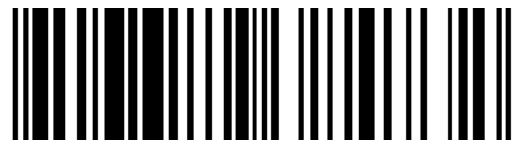

(c) The final best barcode, decodable: 4747379384732

Fig. 7. Comparison of the observed barcode, the initial and final best barcode

After the optimization process, the obtained barcode, shown at the bottom of Figure 7 , is very different from the initial best one, visible on the same figure. It has been successfully decoded and is associated to the code 4747379384732.

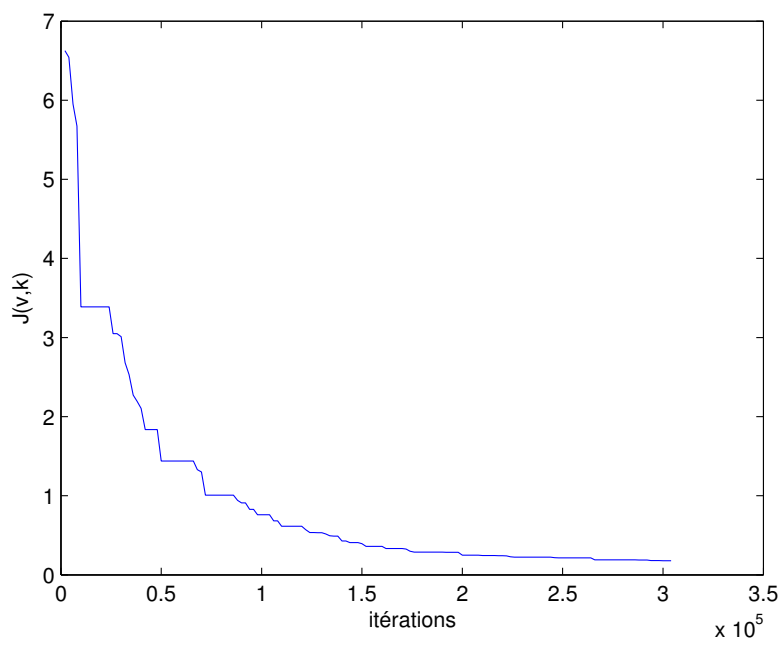

Fig. 8. The convergence history

The convergence history of the optimization process is also depicted in Figure 8 . It shows that the evolutionary algorithm almost performs to achieve a 'perfect' solution, without any a priori information, such as the position of the barcode in the image and starting with a very far initial guess.

A statistical study on a large number of barcodes has also been done, showing that this approach can give positive results, that is a decodable barcode, on a large range of similar signals, even with a very strong noise, a strong blur and with nonuniform illumination.

Example 4: decoding of a real barcode image with blur, noise and nonuniform illumination:

The last example presents the decoding of a real barcode image of poor quality, namely the one depicted in Figure 1 (also visible in Figure 10) and taken with a Nokia N70. The code on the image is undistinguishable because of strong blur. Moreover, the image is also distorted by a strong nonuniform illumination.

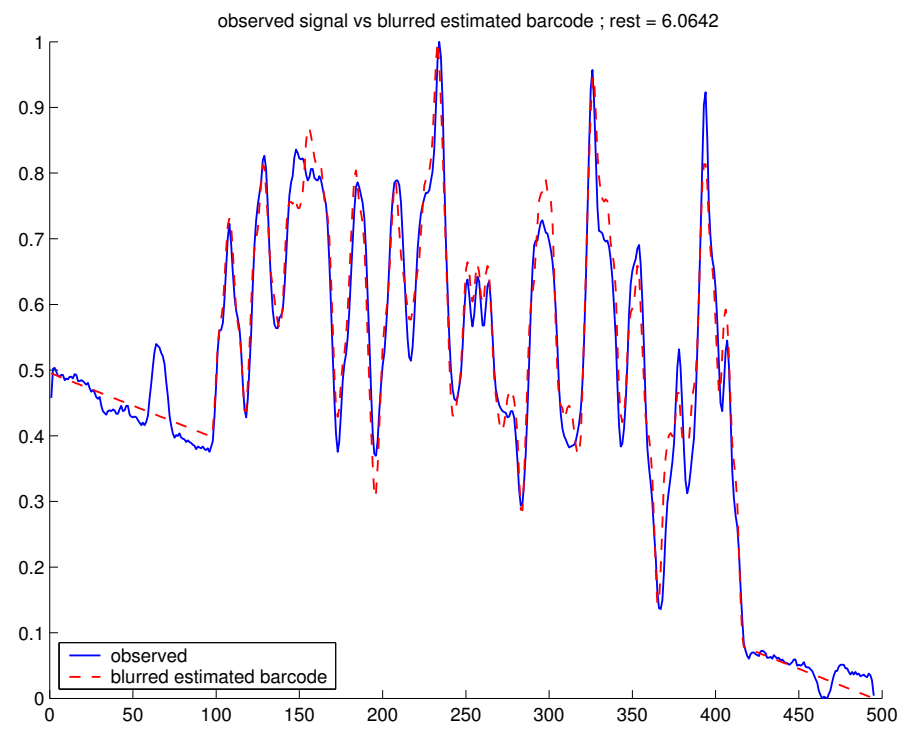

Fig. 9. The observed signal (full line) and the final best signal (dotted line)

By using the same parameters for the genetic algorithm than for the previous example, the obtained best signal is depicted in Figure 9 (red, dotted line) and compared with the observed signal (blue, full line). The matching is almost excellent between both signals. Moreover, the obtained signal, also depicted in Figure 10, has been successfully decoded and is associated with the EAN13 code 4025515825135.

\section{CONCLUSION}

A robust method for blind deconvolution of barcode signals in the presence of blur, noise and with nonuniform illumination is presented here. Based on the resolution of the associated inverse problem with an evolutionary algorithm on a mixed search space, it allows to decode, without any information and in a robust and reproducible way, a very noisy and blurred barcode image. In particular, this approach has been successfully applied to decode barcodes images taken from a camera of very poor quality. 


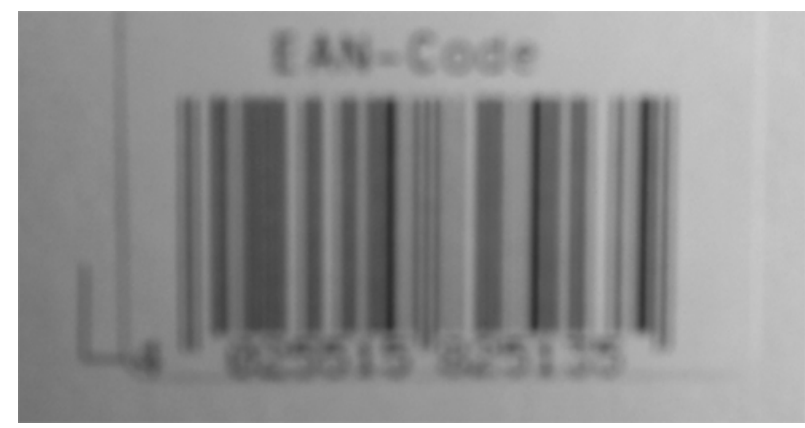

(a) The observed barcode

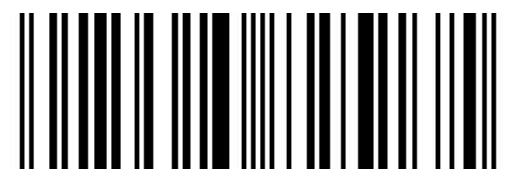

(b) The final best barcode, decodable: 4025515825135

Fig. 10. Comparison of the observed barcode image and the final best barcode

\section{REFERENCES}

[1] Matas J. and Soh L. and Kittler, J.V., AObject recognition using a tag, ICIP 97, pp. 877-880, 1997.

[2] Muniz R. and Junco L. and Otero, A., A robust software barcode reader using the Hough transform, International Conference on Information. Intelligence, and Systems, IEEE, pp. 313-319, 1999.

[3] S. Esedoglu, Blind deconvolution of barcode signals, Inverse Problems, Vol.20, pp.121-135, 2004.

[4] M.D. Sanner Ambient illumination bar code reader, U.S. Patent 4,874,933, 1989.

[5] Shams R. and Sadeghi P., Bar code recognition in highly distorted and low resolution images, International Conf. on Acoustics, Speech and Signal Processing, IEEE, pp. 737-740, 2007.

[6] Zhang L. and Samaras D., Face recognition under variable lighting using harmonic image exemplar, CVPR'03, IEEE, pp. 19-25, 2003.

[7] Wang H. and Li S.Z. and Wang Y., Generalized Quotient Image, CVPR, EEE, pp. 498-504, 2004.

[8] Savvides M. and Vijaya V.K. and Khosla P.L., Corefaces - Robust Shift Invariant PCA based Correlation Filter for illumination Tolerant Face Recognition, CVPR'04, IEEE, Vol.2, 2004.

[9] B. K. Horn, Robot Vision, MIT Press, 1986.

[10] J. Kim and H. Lee, Joint nonuniform illumination estimation and deblurring for bar code signals Optic Express, Vol. 15, issue 22, pp. 14817-14837, 2007.

[11] Goldberg D.E., Genetic Algorithms in Search, Optimization, and Machine Learning, Addison-Wesley, 1989.

[12] Dumas L. and Herbert V. and Muyl F., Hybrid method for aerodynamic shape optimization in automotive industry, Computers ans Fluids, Vol. 33, pp. 849-858, 2004.

[13] Druez B. and Dumas L. and Lecerf N., A fully Adaptive Hybrid Optimization of aircraft engine blades, Journal of Computational and Applied Mathematics, Vol. 232, pp. 54-60, 2009.

[14] Dumas L. and El Alaoui L., How Genetic Algorithms can improve a pacemaker efficiency, Proceedings of GECCO, pp. 2681-2686, 2008. 